\title{
¿QUÉ PIENSAN LOS PROFESORES SOBRE LAS ACTIVIDADES EXPERIMENTALES EN BIOLOGÍA?
}

\author{
WHAT DO THE TEACHERS THINK ABOUT THE EXPERIMENTAL \\ ACTIVITIES IN BIOLOGY?.
}

\author{
Nancy Edith Fernandez-Marchesi*, María Josefa Rassetto**, Emilio Costillo-Borrego ${ }^{\star \star *}$ \\ Instituto de Educación y Conocimiento. Universidad Nacional de Tierra del Fuego, AelAS *, Universidad \\ Nacional de Comahue **, Universidad de Extremadura ***
}

\author{
Correspondencia: Nancy Edith Fernandez-Marchesi \\ Instituto de Educación y Conocimiento. Universidad Nacional de Tierra del Fuego, AelAS \\ Fuegia Basket 251. Ushuaia. (9410). Tierra del Fuego. Argentina \\ Correo: nfernandez@untdf.edu.ar
}

Recibido: 19/09/2018; Aceptado: 27/06/2019

DOI: 10.17398/0213-9529.38.2.143

\section{Resumen}

En este trabajo presentamos resultados acerca del estudio de las concepciones de profesores argentinos de Biología sobre las Actividades Experimentales (AE). Se buscó conceptualizar desde la epistemología, la relevancia histórico-epistemológica de articular la Biología funcional y la Biología evolutiva. El estudio se llevó a cabo desde una perspectiva cualitativa, con base en el análisis de contenido y empleando como técnica de recolección de información un cuestionario validado por expertos para indagar sobre las percepciones respecto a los núcleos temáticos considerados en las $\mathrm{AE}$, los objetivos, las ventajas y las desventajas de dichas actividades. La encuesta se organizó en tres ejes y se distribuyó mediante la lista de correo de la Asociación de Docentes de Ciencias Biológicas de Argentina a profesores de nivel secundario. La muestra quedo conformada por cincuenta y seis profesores. Los resultados indican la predominancia de modelos tradicionales de enseñanza con una imagen clásica de las ciencias considerando a las $\mathrm{AE}$ como actividades necesarias para reforzar teoría con un corte empíricoinductivista. Un número considerable menciona obstáculos institucionales vinculados con la infraestructura disponible en los laboratorios y las condiciones laborales. Los núcleos temáticos preferidos fueron aquellos del campo de la Biología Funcional.

Palabras clave: actividades experimentales, concepciones, profesores, Biología.

\begin{abstract}
In this work we present results about the study of the Argentinean Biology teachers' conceptions about the Experimental Activities (AE). We sought to conceptualize from epistemology, the historical-epistemological relevance of articulating Functional Biology and Evolutionary Biology. The study was carried out from a qualitative perspective, based on the content analysis and using a questionnaire validated by experts as a data collection technique to inquire about the perceptions regarding the thematic nuclei considered in the AEs, the objectives, the advantages and disadvantages of such activities. The survey was organized in three axes and was distributed through the mailing list of the Association of Teachers of Biological Sciences of Argentina to secondary level teachers. The sample was made up of fifty-six professors. The results indicate the predominance of traditional teaching models with a classical image of the sciences considering the EA as necessary activities to reinforce theory with an empirical-inductivist cut. A considerable number mention institutional obstacles linked to the infrastructure available in laboratories and working conditions. The preferred thematic nuclei were those from the field of Functional Biology.
\end{abstract}

Keywords: experimental activities, conceptions, teachers, Biology 


\section{INTRODUCCIÓN}

En primer lugar, consideramos pertinente conceptualizar a qué denominaremos Actividad Experimental (AE) en Biología. Tomando las palabras de Hacking (1996) entendemos que en una $A E$ deben identificarse: la posibilidad de aislar o controlar el fenómeno biológico, la observación orientada ya sea con o sin instrumentos especializados, y la intervención sobre el fenómeno que es el momento clave de la experimentación, cuando el observador manipula diferentes variables con algún propósito.

A pesar de lo mencionado, no podemos dejar de señalar que en el campo de la epistemología de la Biología llama la atención los contrastes existentes entre la Biología experimental de los laboratorios y la Biología observacional de campo (Caponi, 2001, 2003). Han sido Jacob (1973) y Mayr (1988) quienes han mostrado la posibilidad y la relevancia histórico-epistemológica de articular estas distinciones insertándolas entre dos vastos dominios de indagación científica, la Biología funcional y la Biología evolutiva (Caponi, 2003). La primera, siguiendo procedimientos experimentales semejantes a los de la Química y la Física, se ocupa del estudio de las causas próximas. La segunda, mientras tanto, sería esa otra Biología ocupada en indagar, recurriendo, básica pero no exclusivamente, a evidencia observacional, a métodos comparativos y a inferencias históricas, causas remotas que, actúan a nivel de las poblaciones (Caponi, 2001).

Esta diferenciación genera como consecuencia que los procedimientos experimentales de la Biología funcional puedan asimilarse a los de la Química y/o la Física, pero en la Biología evolutiva casi todos los fenómenos son explicados por inferencias basadas en estudios comparativos con descripciones muy minuciosas (Mayr, 1998). Esta distinción genera un correlato que impacta en la enseñanza en el aula, dado que, como veremos más adelante, el profesorado de Biología se inclina más por las AE incluidas en la Biología funcional que las incluidas en la Biología evolutiva.

\section{Las actividades de laboratorio y su enseñanza}

La enseñanza de las AE debe proporcionar la oportunidad de hacer buenas preguntas sobre la naturaleza, practicar el reconocimiento de regularidades, diversidades y puntos comunes entre las observaciones, reconocer los problemas, comprender los métodos experimentales, organizar e interpretar los datos, comprender la relación de los hechos con la solución de los problemas, planificar experimentos para probar hipótesis y hacer generalizaciones y suposiciones. (Kirschner, 1992)

Para ello, es interesante destacar que el estilo didáctico utilizado por el profesorado cuando "va" al laboratorio, es en gran medida una de las causas que promueven las visiones distorsionadas de la ciencia. La enseñanza tradicional del laboratorio ha conducido a una comprensión inadecuada de la naturaleza de la ciencia, debido a que se ha basado en una concepción empírico-inductivista del "método científico", derivada del positivismo baconiano, entendido como un proceso de pasos característicos casi mecánicos de la actividad científica: observación y experimento, generalización inductiva, hipótesis, verificación, prueba y contraprueba y conocimiento objetivo, lo cual se asocia a una creciente objetividad y neutralidad conceptual del científico. (Carles, Payá, y Valdés, 2005; Kirschner, 1992)

Según Kirschner (1992), el trabajo experimental se debe utilizar para enseñar y aprender la estructura sintáctica de una disciplina, más que su estructura sustantiva. La estructura sintáctica de la ciencia, promueve en los estudiantes el desarrollo de habilidades de razonamiento y pensamiento para utilizarlas en diversos contextos (Kirschner, 1992). Este es el 
nudo del problema: las prácticas se usan muy a menudo para enseñar, afirmar o ilustrar la estructura sustantiva (conceptos), mientras que son más adecuadas para transmitir su estructura sintáctica (habilidades de pensamiento).

Este autor, plantea además, que los laboratorios húmedos ${ }^{1}$ son el tipo más común encontrado en los currículos de ciencias y los clasifica en formales, experimentales y divergentes (Tabla 1).

Tabla 1:

Tipos de laboratorios según Kirschner (1992)

\begin{tabular}{|c|c|}
\hline $\begin{array}{l}\text { Laboratorio formal } \\
\text { (tradicional, } \\
\text { estructurado, } \\
\text { laboratorio } \\
\text { convergente, de } \\
\text { libro de cocina) }\end{array}$ & $\begin{array}{l}\text { Funciona principalmente para verificar las leyes, los principios, los conceptos y los } \\
\text { hechos enseñados en conferencias y dados en libros de texto. } \\
\text { Se le dice al estudiante exactamente qué hacer. } \\
\text { Tiempo rígidamente establecido } \\
\text { Debe llegar a los resultados esperados (implícitamente implícitos o explícitamente } \\
\text { indicados). } \\
\text { Los informes de laboratorio realizado por los estudiantes son vistos como simples } \\
\text { obligaciones y guardan poca semejanza con los informes elaborados por científicos } \\
\text { profesionales. }\end{array}$ \\
\hline & $\begin{array}{l}\text { estudiante aquí se enfrenta a problemas de experimentación destinado a desafiar } \\
\text { comprensión y la creatividad sin ser tan complejo como para ser imposible de } \\
\text { solver. }\end{array}$ \\
\hline $\begin{array}{l}\text { experimental } \\
\text { (abierto, inductivo, } \\
\text { descubrimiento) }\end{array}$ & $\begin{array}{l}\text { Las instrucciones son generales, excepto en los casos en que un error sería costoso } \\
\text { o peligroso. } \\
\text { Experimentos diseñados para mostrar las limitaciones de la teoría, mientras que al } \\
\text { mismo tiempo muestra la necesidad de teoría y la interdependencia compleja entre } \\
\text { lo teórico y lo experimental. }\end{array}$ \\
\hline $\begin{array}{l}\text { En el laboratorio } \\
\text { divergente }\end{array}$ & $\begin{array}{l}\text { Partes del experimento están predeterminadas y comunes para todos los } \\
\text { estudiantes. } \\
\text { Existen muchas direcciones posibles en las que el experimento pueda desarrollarse } \\
\text { después de la etapa inicial. }\end{array}$ \\
\hline
\end{tabular}

Con respecto a las diversas modalidades (o tipos) de actividades o estrategias llevadas a cabo en el laboratorio han surgido diversas voces en la literatura, algunos autores (Hodson, 1994; Miguens y Garrett, 1991) han mencionado que el enfoque por descubrimiento (muy popular en la década del '60), resultó un fracaso, por su fuerte arraigo inductivista y por ser epistemológicamente equivocado, psicológicamente erróneo y pedagógicamente impracticable además de que el procedimiento de descubrimiento cae en la trampa de considerar la observación como objetivo y punto de partida del método científico.

Por otro lado, numerosos trabajos (de Pro Bueno, Sánchez Blanco, y Valcárcel Pérez, 2008; Fernández-Marchesi, 2014; Rivarosa y Astudillo, 2013) han planteado que las actividades de laboratorio que siguen protocolos tipo "receta" (estilo formal - académico) promueven escasas habilidades, y están alejadas de las propuestas epistemológicas y didácticas de la literatura especializada.

Por ello, las actividades de laboratorio deben promover procesos cognitivos que faciliten la comprensión de un sistema explicativo sobre un hecho o fenómeno más allá que la "simple observación y demostración", y de la manipulación de elementos y materiales de un laboratorio o campo. Este enfoque implica problematizar, promover la elaboración de diseños, plantear preguntas que cuestionen el sentido común, análisis de variables observables y teóricas, atender al error experimental, argumentar y reflexionar sobre el proceso y su resultado (metacognición). (Rivarosa y Astudillo, 2013) 


\section{Epistemologías personales de los profesores}

La investigación sobre las concepciones y la práctica de los profesores constituyen una línea fundamental en la agenda de investigación en didáctica de las ciencias (Freitas, Jiménez, y Mellado, 2004; Mellado, Ruiz, Bermejo, y Jiménez, 2006; Tobin, Tippins, y Gallard, 1994; Vázquez Bernal, Jiménez Pérez, y Mellado, 2007). Estas concepciones y marcos epistemológicos construidos en la formación inicial impactan en las prácticas de clase cuando diseñan las actividades que realizarán los estudiantes.

Los futuros profesores poseen concepciones sobre los procesos de enseñanzaaprendizaje, en general, y sobre las actividades experimentales, en particular. Estas han generado actitudes fruto de su experiencia como estudiante, y como parte de la continua evolución que sufren las ideas en la etapa escolar hasta su desarrollo profesional. Estas concepciones pedagógicas son muy estables y apenas cambian durante el programa de formación inicial (Astudillo, Rivarosa, y Ortiz, 2011; Bachelard, 1973; Carrascosa, Martínez Torregosa, Furió Más, y Guisasola Aranzábal, 2008; Mellado y col., 2006; Perrenoud, 2004; Porlán y col., 2010; Porlán, Rivero García, y Martín del Pozo, 1998). Por tanto, todas estas creencias y marcos epistemológicos deben ser considerados y constituir el punto de partida del conocimiento experto que se implemente durante los programas formativos de los profesores de ciencias.

Estas epistemologías personales, que orientan y condicionan los procesos de adquisición de conocimientos significativos en el aula, son reconstruidas a lo largo de la formación docente y están constituidas por un entramado de conocimientos empíricos, simbólicos y afectivos, que ofrecen resistencia a ser modificadas, modelan la lectura de la realidad y están caracterizadas como estables, regulares y generalizables (Perrenoud, 2004; Pozo Municio y col., 2006; Rivarosa y Astudillo, 2013). Además, repercuten en el diseño y realización de las actividades experimentales que desarrollarán como profesores, afectando a los aprendizajes de sus futuros estudiantes.

Porlán y Martín del Pozo (1996), expresan la importancia de conocer las concepciones científicas del profesorado, porque entre otras cuestiones:

- tienen cierta relación con el modelo de enseñanza que se lleva a cabo y con lo que se entiende por enseñar.

- guardan cierta coherencia con las concepciones que se refieren a cómo aprenden ciencias los alumnos.

- influyen en las concepciones científicas del alumnado y contribuyen a formar y/o reforzar la imagen de la ciencia del público en general.

No podemos obviar que el conocimiento didáctico del contenido (Shulman, 1986) es lo que guía las prácticas docentes en el aula, pero no como una mezcla estática de los diferentes conocimientos académicos, sino que, a partir de la reflexión-en-la-acción y de la reflexiónsobre-la-acción de enseñar (Perrenoud, 2004), el profesor transforma e integra los distintos conocimientos, en un proceso activo y dinámico. El conocimiento didáctico del contenido se genera y evoluciona a partir de los propios conocimientos académicos, filtrados por las concepciones, valores, actitudes y emociones, pero requiere de la implicancia y reflexión personal sobre el proceso de enseñanza de la materia específica, en contextos escolares concretos. (Garritz y Mellado, 2014; Mellado y col., 2014; Melo Niño, Cañada Cañada, Mellado, y Buitrago, 2016) 
Esta idea supone promover situaciones sobre contenidos específicos y contextualizados que permitan a los futuros profesores reflexionar expresamente sobre cómo se desarrollarían dichos contenidos en la práctica docente, tomando como punto de partida el análisis de las propuestas o actuaciones de los formadores de formadores. (Martínez-Aznar, RodríguezArteche, y Gómez Lesarri, 2017). Con la experiencia profesional, a través de los años de enseñanza, el profesor de ciencias integra en una estructura única los diferentes componentes del conocimiento, desarrollando su propio estilo de enseñanza. Pensar en el docente como un didacta de autor (Anijovich, Cappelletti, Mora, y Sabelli, 2009; Litwin, 2000) que diseña situaciones educativas, implica ubicarse en una perspectiva reflexiva que considera la tarea educativa como variables multidimensionales, contextuales e ideológicas y, a la tarea cotidiana del enseñar, como una hipótesis de trabajo, imperfecta, falible, creativa y provisional.

Sin embargo en palabras de Perrenoud (2004) si esta práctica reflexiva no es ni metódica, ni regular, no genera una toma de conciencia y un cambio. Una práctica reflexiva metódica se inscribe en el tiempo de trabajo como una rutina. Pero no como una rutina somnífera sino como un estado de alerta permanente para lo cual necesita una disciplina y métodos, para observar, memorizar, escribir, analizar a posteriori, comprender y escoger nuevas opciones. (Perrenoud, 2004)

\section{OBJETIVOS}

La finalidad del estudio fue indagar sobre las concepciones que poseen un grupo de profesores argentinos de Biología sobre las actividades experimentales. Dentro de este objetivo global hemos considerado otros más específicos. Estos fueron:

- Identificar y caracterizar sus concepciones sobre los objetivos, las ventajas y las desventajas de las AE

- Analizar qué núcleos temáticos de la Biología (Fernández-Marchesi, 2014) eligen los profesores cuando realizan $\mathrm{AE}$

- Comprender la relación teoría-práctica en la implementación de las AE en sus clases.

\section{METODOLOGÍA}

Se aplicó un cuestionario validado por expertos (Anexo I) elaborado en un formulario de Google. Para validarlo, se diseñó un primer borrador y se envió a investigadores expertos quienes observaron y sugirieron modificaciones a las preguntas. La encuesta se organizó en tres ejes: a) datos personales (edad, titulación, años de antigüedad docente, colegio donde trabaja); b) preguntas en relación a las $A E ; c)$ redacción de un guion de una práctica experimental. En este trabajo se presentan solamente las respuestas de los ejes a) y b).

Se envió dos $^{2}$ veces mediante la lista de correo de la Asociación de Docentes de Ciencias Biológicas de Argentina ${ }^{3}$ entre los meses de julio y septiembre de 2017 a profesores de nivel secundario de Argentina.

Se recogieron 67 (sesenta y siete) respuestas. Una vez analizadas, se tomaron aquellas que pertenecían solamente a profesores ${ }^{4}$. La muestra quedo conformada por 56 (cincuenta y seis) profesores. Para su mejor análisis se numeraron y se sistematizaron las respuestas en diversas hojas de una planilla de cálculo.

Las respuestas se analizaron mediante el método de análisis de contenidos (Bardin, 1996). Este método consiste en la manipulación de mensajes (el contenido y la expresión de ese contenido), para inferir lo que está implícito en el documento. Principalmente se utiliza la 
lectura como técnica para la recolección de los datos, una lectura sistemática, replicable y validada. Se buscaron indicadores que permitieron inferir lo que estaba implícito en el mensaje.

En el caso del primer objetivo las respuestas obtenidas se sistematizaron en una red sistémica (Bliss, Monk, y Ogborn, 1983). Una red sistémica es un conjunto estructurado de opciones interdependientes que muestra en su estructura un patrón de rasgos descriptivos relacionados agrupados mediante etiquetas que las explican (Bliss, Ogborn, y Grize, 1979). La red no describe los datos, sino una interpretación de los mismos con el propósito de ordenar la información.

Las categorías inferidas fueron:

I) contenidos: conceptos, procedimientos y actitudes. (de Pro Bueno, 1998 y Martínez-Aznar y Ibáñez Orcajo 2006)

II) gestión: de aula y de la institución. (Fernández-Marchesi y Martínez-Aznar, 2017)

Para la construcción de la Red Sistémica se tomó como base la presentada por Fernández Marchesi y Martínez-Aznar (2017). Se la adaptó al contexto argentino y se adecuaron algunas de las categorías a las respuestas recogidas de los cuestionarios (Figura 2). Se contabilizaron y se obtuvieron los porcentajes de cada uno de los enunciados para cada categoría y subcategoría

Para el segundo objetivo se utilizaron como base los núcleos temáticos propuestos por Fernández Marchesi (2014) y se definieron otros según los contenidos mencionados por los profesores y profesoras encuestados. Los Núcleos Temáticos corresponden a los temas generales de estudio de la Biología; agrupan conceptos y grandes ideas de las Ciencias Biológicas; desde el ámbito disciplinar, un núcleo temático incluye contenidos de diversas sub disciplinas. Luego se construyeron gráficos que permitieron visualizar las respuestas. (Fernández-Marchesi, 2014)

En el tercer objetivo se buscó identificar qué tipo de relación mencionaban los docentes entre las "clases teóricas" y las "clases prácticas".

\section{RESULTADOS Y DISCUSIÓN}

\section{Contexto}

Para contextualizar el estudio, se describen las características de: titulación de base, edad y años en el desempeño como profesores.

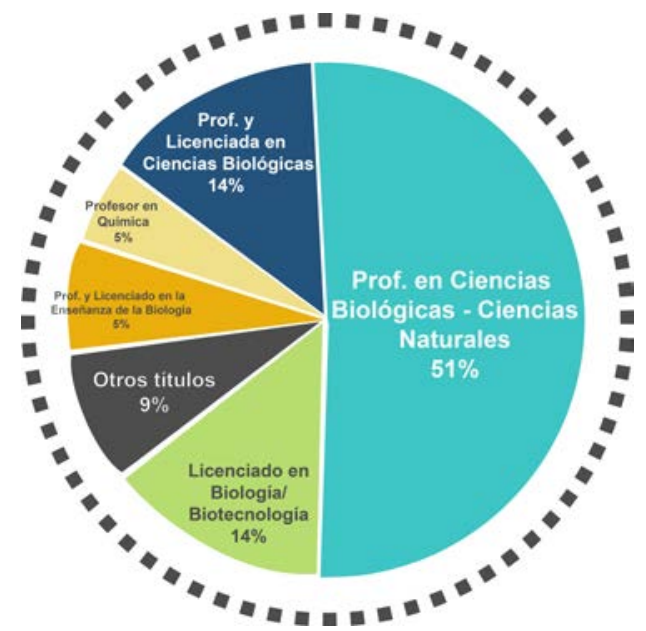

Figura 1: Titulación de los profesores encuestados 
En Argentina, la formación de profesores se realiza en Institutos de Educación Superior, existiendo más de 1000 en todo el país (gestión pública y privada). Además, pueden obtener la titulación de profesor quienes, realizando una carrera de Licenciatura en Biología (Biólogo), además curse y apruebe materias de corte pedagógico. Con ello obtiene el título de Profesor y Licenciado en Biología. También, y dado el déficit de profesores existentes pueden ejercer en el nivel secundario, profesionales (médicos, abogados, enfermeros, técnicos, ingenieros, etc) que cuenten con formación pedagógica ${ }^{5}$ o no.

Más de la mitad de los encuestados poseen título de profesor (72\%). Entre ellos, un pequeño porcentaje posee además otro título de grado: Licenciado en Biología o Licenciado en Enseñanza de la Biología. En el primer caso, es un título de grado universitario (4 años) cuya formación se basa fundamentalmente en la Biología y sus disciplinas afines. En el segundo caso, corresponde también a un título de grado universitario obtenido a través de un ciclo de complementación curricular ( 2 años) pero cuyo énfasis está puesto en la enseñanza de la Biología (la didáctica y las teorías curriculares) (Figura 1).

Con respecto a la edad el promedio es de 39 años siendo menores de 40 años el $67 \%$ de los encuestados.

Queda en evidencia que no se trata de profesores muy jóvenes ${ }^{6}$ (solo el 21\% posee menos de 30 años), pero sí, la gran mayoría declara pocos años dando clases (el 68\% declara menos de 10 años - no obstante, el promedio general es de 11 años). Esto supone dos cosas, o que inician su labor como docente luego de varios años de egresar del Instituto de Formación Docente o que inician sus estudios luego de varios años de finalizar el nivel secundario.

\section{Concepciones sobre los objetivos, las ventajas y las desventajas de las AE}

Los resultados indican que los profesores poseen en general una visión tradicional acerca de las actividades experimentales ya que la mayoría las considera como actividades que permiten "aplicar" contenidos. Se destacan tres ideas con mayor énfasis: "reforzar contenidos" (41\%), permitir el paso de lo "abstracto a lo concreto" (61\%) e "identificar e interpretar" (36\%) la teoría mediante la implementación de actividades experimentales (Figura 2). Se observa que un $21 \%$ reconoce que las $A E$ pueden ser útiles para promover aprendizaje significativo. Es llamativo que apenas un $5 \%$ indica que es posible vincular las AE con la "vida cotidiana", lo cual permite inferir que su realización es descontextualizada y no promueven en enfoque Ciencia Tecnología y Sociedad (CTS). 


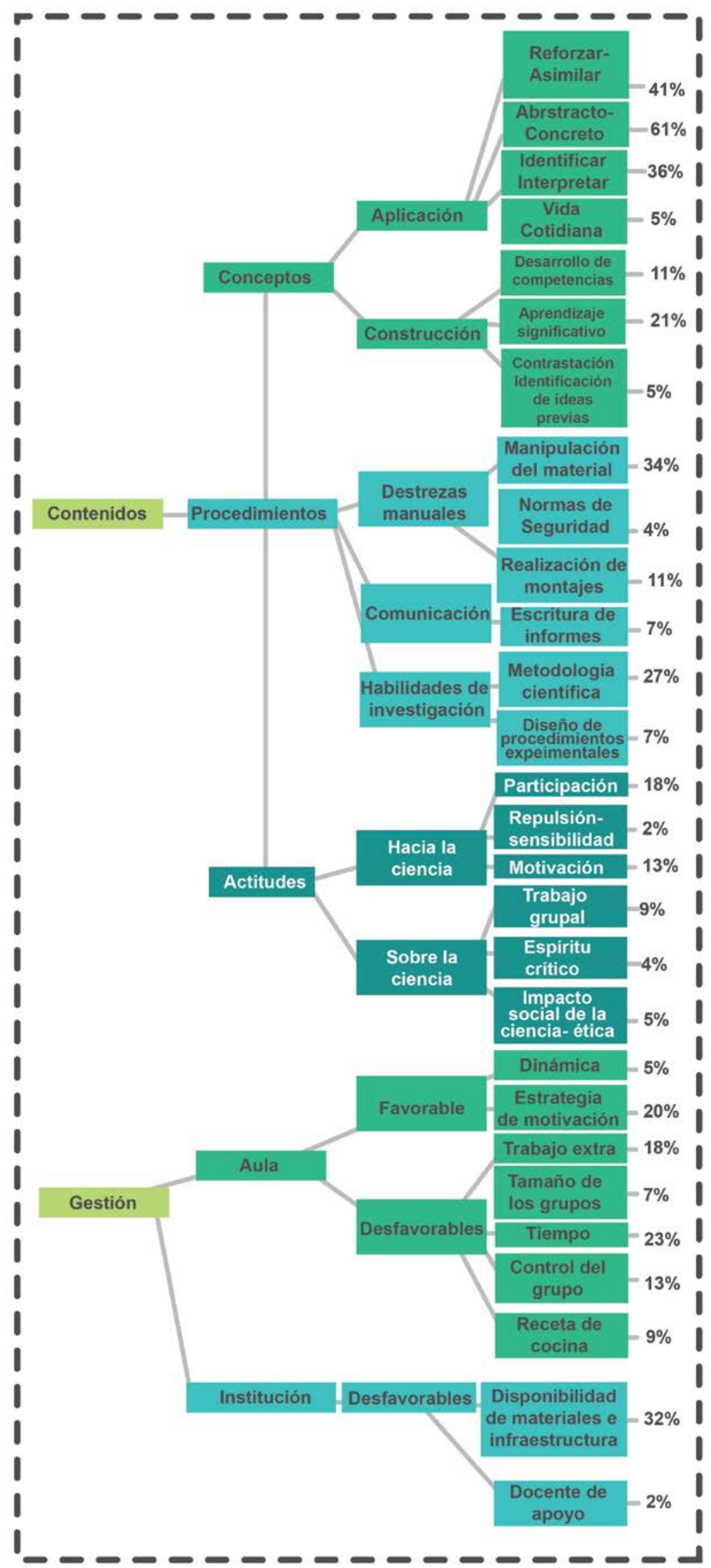

Figura 2: Red sistémica de las categorías establecidas sobre las concepciones de profesores de Biología de Argentina 
Con respecto a los contenidos procedimentales, la mayoría se inclina por "manipulación de materiales" (34\%) e implementación de "metodología científica" (27\%). Es notorio que sólo el $7 \%$ le otorga relevancia a la comunicación científica dentro de las AE (Figura 2).

Estos resultados coinciden con los alcanzados por autores como Cortés Gracia y de la Gándara Gómez (2006); do Carmo Galiazzi y col. (2001); Fernández Marchesi, CostilloBorrego, y Rivarosa (2016); Fernández Marchesi, Martínez-Aznar, y Costillo-Borrego (2017); Fernández Marchesi y Martínez-Aznar (2017) e Insausti y Merino (2000), todos ellos indican que las actividades experimentales son utilizadas como refuerzo de la teoría, aplicación de conceptos en la práctica, "ver" en la práctica lo estudiado en la teoría, recolección de datos, realización de cálculos, aplicación del método científico y aprendizaje de técnicas de laboratorio. A partir de estos datos se infiere una concepción de ciencia consolidada de carácter empirista e instrumental.

Con respecto a las preguntas sobre ventajas y desventajas de la implementación de AE en las clases de ciencias, las menciones relacionadas con las actitudes hacia la ciencia y sobre la ciencia representa un 31\%, repartidas entre actitudes como la "motivación y la "participación" Estos resultados son bastante semejantes a los expresados en otros trabajos en los que se encuestó a futuros profesores (Fernández-Marchesi y Martínez-Aznar, 2017; FernándezMarchesi y col., 2017). Podría deberse a que las respuestas están vinculadas con los recuerdos y emociones de la propia biografía académica y escolar (Costillo-Borrego, Borrachero Cortés, Brígido Mero, y Mellado, 2013). Esto también indica una visión tradicional, en otras actividades prácticas muchos profesores tradicionalmente se decantan sobre todo por el aspecto positivo de las salidas sobre las actitudes de los alumnos (Bitgood, 1989; Meredith, Fortneer, y Mullins, 1997), sosteniendo que son sobre el campo de las emociones de los alumnos donde las salidas a la naturaleza inciden en mayor medida. (Meredith y col, 1997)

En cuanto a la categoría de gestión, el $20 \%$ atribuyen como aspecto favorable a las prácticas experimentales las "estrategias de motivación" que poseen (Figura 2). Sobresalen expresiones tales como:

"Motivación del alumno, rol del alumno activo" (E16);

"Despierta interés. Acapara la atención" (E17);

"La motivación que genera en los estudiantes este tipo de actividades creo que es una de las ventajas por excelencia. Esto permite una mejor predisposición en el encuentro con el nuevo conocimiento a construir" (E19).

También en esta categoría surgen como posibles dificultades aspectos relacionados con el "trabajo extra del docente" (18\%) y "tiempo" (23\%) en términos de la preparación previa que requiere realizar una $\mathrm{AE}$ en las clases y en el desarrollo de los contenidos de la misma.

"Se emplean más clases, para temas que con una clase expositiva se abordaría en tres bloques de 40' cada uno" (E2);

"A veces se insume un tiempo que es difícil ajustar a la estructura rígida escolar" (E8); "Poder realizar un seguimiento minucioso de los avances que desarrollan los estudiantes a través de la práctica y el acompañamiento a cada uno de ellos de manera profunda" (E13).

Un número significativo mencionan dificultades institucionales (32\%) vinculados con la disponibilidad de los materiales o infraestructura disponible (Figura 2) siendo coincidente a lo expresado por otros autores (Fernández Marchesi y col., 2017). Este factor merecería ser estudiado con mayor profundidad y amplitud dado que en general la mayoría de las investigaciones sólo refieren a lo que "sucede" en el laboratorio y no incluyen en el análisis los 
factores institucionales ligados a la práctica docente: burocracia, seguridad de estudiantes y docentes en el laboratorio o en el campo, la presencia o no de un jefe de trabajos prácticos, la continuidad del cargo del ayudante de laboratorio, las regulaciones del trabajo docente, la distribución de los tiempos escolares (clases de 40 minutos), entre otros motivos.

\section{Núcleos temáticos preferidos por los docentes para realizar $\mathrm{AE}$}

Los docentes han elegido una amplia variedad de núcleos temáticos a la hora planificar sus $A E$, si bien algunos de ellos concentran gran parte de las propuestas. La gran mayoría de los contenidos indicados corresponden al núcleo temático de "Biología celular y molecular" (21 menciones ${ }^{7}$ ) siguiendo en orden de mención "técnicas y uso de instrumentos" (8 menciones). Aquí queda evidenciado lo que fue mencionado anteriormente: el profesorado se inclina por los temas incluidos en la Biología funcional (Caponi, 2003). Suponemos que por la posibilidad de realizarlas en el aula o en el laboratorio ya que serían AE sencillas, cortas, inmediatas que pueden resolverse en una o dos clases y no requieren de estudios a largo plazo.

\begin{tabular}{|c|c|}
\hline GENERALES & \\
\hline Técnicas y uso de instrumentos & 8 \\
\hline No especifica & 8 \\
\hline FÍSICO-QUIMICA & \\
\hline Sistemas materiales & 4 \\
\hline Reacciones químicas & 3 \\
\hline Mezclas & 1 \\
\hline Soluciones & 2 \\
\hline Energía & 2 \\
\hline BIOLOGIA & \\
\hline Genética & 1 \\
\hline Biodiversidad & 3 \\
\hline Ecología & 3 \\
\hline Biologia humana & 1 \\
\hline Biología celular y molecular & 21 \\
\hline
\end{tabular}

Figura 3: Distribución de los contenidos en núcleos temáticos elegidos por los docentes a la hora de planificar las Actividades Experimentales (AE)

\section{Relación teoría-práctica en la implementación de AE}

En el caso del tercer objetivo en su mayoría, las afirmaciones se deciden por dedicar una clase a explicar los contenidos teóricos seguida de otra de carácter práctico (40\%). Este lugar otorgado a las AE responde al modelo tradicional identificado anteriormente. Sólo un $8 \%$ plantearon un modelo diferente sugiriendo la resolución de problemas como estrategia.

"Se trabajaría con una situación problemática y luego los estudiantes elaborarían su propia experiencia que luego llevarían a cabo." (E42); 
"Actividades de indagación, proponer un problema a resolver en el laboratorio y luego presentación de conclusiones" (E16).

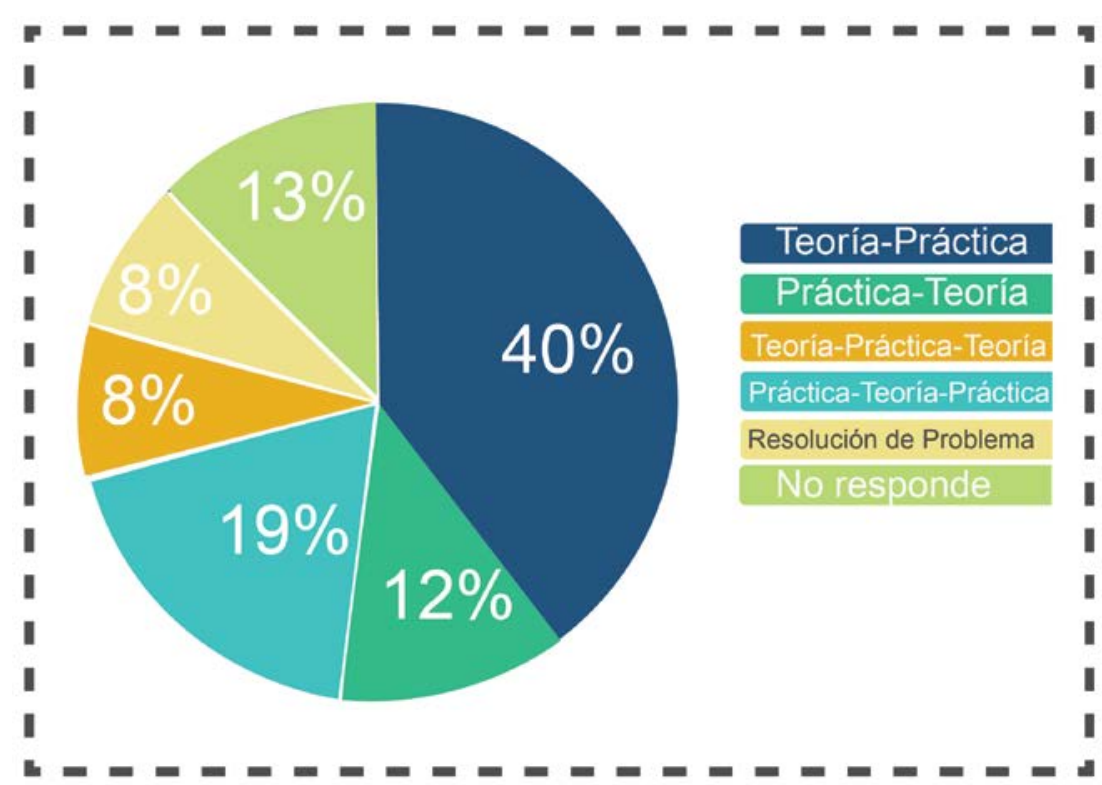

Figura 4: Relación de la Actividades Experimentales con la teoría, se expresan los porcentajes de las secuencias temporales propuestas.

Un $21 \%$ de los sujetos plantearon algún tipo de interacción teoría - práctica. En este caso las valoraciones de las actividades de laboratorio aparecen diferenciando entre clases teóricas (rígidas y aburridas) y prácticas-procedimentales (dinámicas, participativas y flexibles). La idea generalizada es que la ciencia es algo a lo que se accede a través de un estudio adecuado de los contenidos conceptuales que trae "de regalo" el aprendizaje de los contenidos procedimentales mediante las clases prácticas. (do Carmo Galiazzi y col., 2001; Insausti y Merino, 2000)

\section{Implicaciones didácticas para la formación de formadores}

El debate sobre la relación teoría- práctica o sobre el papel de la teoría versus el papel del experimento es antiguo en filosofía de la ciencia. Hacking (1996) da un giro en la filosofía de la ciencia reivindicando el papel del experimento en la práctica científica. Su pretensión no es eliminar las teorías como representación del mundo sino abandonar el esquema jerárquico en el que el experimento está al servicio de la teoría.

Este autor es especialmente interesante para la enseñanza de las ciencias porque pone el experimento al mismo nivel que la teoría. Hay un equilibrio entre ellos. Reconoce que podemos encontrarnos casos, tanto en que el experimento prima sobre la teoría, como casos en que sería difícil hacer avanzar la ciencia sin tener en cuenta determinados modelos teóricos (García Arteaga y Estany, 2010).

En coincidencia con Giraldo Mejía (2016), los resultados analizados, permiten inferir que en relación al papel que se le otorga a la experimentación desde la imagen "clásica" de las ciencias, se encontraron dos posturas. Una de ellas, concibe la experimentación como el único medio para validar, confirmar o contrastar teorías. Los fenómenos son considerados accesibles a través de los sentidos, de modo que la observación empírica lleva de forma objetiva a enunciados observacionales verdaderos (Quesada Blázquez, 2006). La concepción "clásica" o "concepción heredada de las ciencias", es el legado más representativo que el positivismo y el 
empirismo lógico han dejado en la enseñanza de las ciencias naturales. (Caponi, 2003; Hacking, 1996; Iglesias, 2004; Romero Chacón y col., 2017)

La segunda postura, que se basa en la perspectiva empiro-inductivista, afirma que el experimento es la base exclusiva a partir del cual se fabrican los postulados teóricos y, por consiguiente, la única manera de construir conocimiento científico. (García Arteaga, 2011)

A pesar de las diferencias entre estas concepciones, ambas toman como sustento la evidente separación entre la teoría y la práctica, "una separación entre el mundo de las ideas y el mundo de lo sensible, y se considera que, a través del experimento, de alguna manera se establece un nexo entre estos dos mundos" (Malagón y col., 2011, p.19 citado por Giraldo Mejía, 2016)

Es fundamental considerar la importancia de una formación de profesores que provoque el cambio de las concepciones sobre los objetivos de las AE y supere estas visiones que se manifiesta en las aulas de ciencias (do Carmo y col., 2001). Dicha superación exige el establecimiento de puentes entre las creencias que tienen los sujetos sobre la actividad experimental y las prácticas docentes, y las que se proponen desde la formación. (Jiménez y Oliva, 2016)

Por ello, es conveniente salir de aquellos enfoques de formación que se limitan a transmitir principios educativos o a dar a conocer modelos ideales. Interesa conocer cómo progresa el conocimiento del profesorado en escenarios formativos que promueven la reflexión explícita sobre problemas específicos de la enseñanza de ciencias, en el marco de actividades de creación y fundamentación de hipótesis didácticas (Astudillo y col., 2011; Astudillo, Rivarosa, y Ortiz, 2014; Porlán y col., 2010, 2011; Porlán, Rivero García, y Martín del Pozo, 1997). Como explica Perrenoud (2004), una actitud reflexiva no se construye espontáneamente, corresponde especialmente a la formación, inicial desarrollarla y facilitar los conocimientos y el saber hacer correspondiente. Para ello, es importante que la formación desarrolle las capacidades de auto-socio-construcción del habitus, del saber hacer, de las representaciones y de los conocimientos profesionales. De este modo, permitirá una relación con la propia práctica y con uno mismo, una postura de autobservación, de autoanálisis, de planteamiento y de experimentación, y facilitará una relación reflexiva con lo que hacemos.

Para que un cambio profundo sea posible es fundamental reflexionar y tomar conciencia de los esquemas de acción, las creencias, los supuestos que subyacen en el quehacer cotidiano del aula y es necesario reestructurarlos y modificarlos estratégicamente para actuar en situaciones futuras. Como reflexionar es reflejarse, mirar atrás, mirarse en el otro, para luego proyectar, necesitamos una pausa en ámbitos colectivos para que ocurra la toma de conciencia y la reorganización del hacer (Anijovich y col., 2009). Tal como está organizado el sistema escolar en Argentina a la fecha es bastante improbable que puedan darse estos espacios de reflexión

\section{REFERENCIAS}

Anijovich, R., Cappelletti, G., Mora, S., y Sabelli, M. J. (2009). Transitar la formacion pedagogica. Buenos Aires: Paidós.

Astudillo, C., Rivarosa, A., y Ortiz, F. (2011). Formas de pensar la enseñanza en ciencias. Un análisis de secuencias didácticas. Revista Electrónica de Enseñanza de Las Ciencias, 10, 567-586.

Astudillo, C., Rivarosa, A., y Ortiz, F. (2014). Reflexión docente y diseño de secuencias didácticas en un contexto de formación de futuros profesores de ciencias naturales. Perspectiva Educacional, 53(1), 130-144.

Bachelard, G. (1973). Epistemología. Barcelona, España: Editorial Anagrama. 
Bardin, L. (1996). Análisis de contenido. Madrid: Akal.

Bitgood, S. (1989). School Field Trips: An Overview. Visitor Behavior, IV(2), 3-6.

Bliss, J., Monk, M., u Ogborn, J. (1983). Qualitative data analysis for educational research: A guide to uses of systemic networks. Routledge Kegan y Paul.

Bliss, J., Ogborn, J., u Grize, F. (1979). The Analysis of Qualitative Data. European Journal of Science Education, 1(4), 427-440.

Caponi, G. (2001). Biología funcional vs. Biología evolutiva. Episteme: Filosofia e Historia Das Ciencias, (12), 23-46.

Caponi, G. (2003). Experimentos en Biología Evolutiva: ¿Qué tienen ellos que los otros no tengan? Episteme: Filosofia e Historia Das Ciencias, (16), 61-97.

Carrascosa, J., Martínez Torregrosa, J., Furió Más, C., y Guisasola Aranzábal, J. (2008). ¿Qué hacer en la formación inicial del profesorado de ciencias de secundaria? Revista Eureka Sobre Enseñanza y Divulgación de Las Ciencias, 5(2), 118-133.

Cortés Gracia, A., y de la Gándara Gómez, M. (2006). La construcción de problemas en el laboratorio durante la formación del profesorado: una experiencia didáctica. Enseñanza de Las Ciencias, 25(3), 435-450.

Costillo-Borrego, E., Borrachero Cortés, A., Brígido Mero, M., y Mellado, V. (2013). Las emociones sobre la enseñanza-aprendizaje de las ciencias y las matemáticas de futuros profesores de Secundaria. Revista Eureka sobre Enseñanza y Divulgación de las Ciencias, 10(Extraordinario), 514-532.

de Pro Bueno, A. (1998). ¿Se pueden enseñar contenidos procedimentales en las clases de ciencias? Enseñanza de Las Ciencias, 16(1), 22-41.

de Pro Bueno, A., Sánchez Blanco, G., y Valcárcel Pérez, M. (2008). Análisis de los libros de texto de Física y Química en el contexto de la Reforma LOGSE. Enseñanza de Las Ciencias, 26(2), 193210.

do Carmo Galiazzi, M., de Barros Rocha, J., Schmitz, L., Langoni de Souza, M., Giesta, S., y Peres Gonçalves, F. (2001). Objetivos das atividades experimentais no ensino medio: a pesquisa coletiva como modo de formaçao de professores de Ciencias. Ciência y Educação, 7(2), 249-263.

Fernández-Marchesi, N. (2014). Los trabajos prácticos de laboratorio de Biología en los libros de texto de Ciencias Naturales para el Nivel Secundario utilizados en la Ciudad de Ushuaia. Tesis de Maestría. Facultad de Ingeniería. Universidad Nacional del Centro de la Provincia de Buenos Aires.

Fernández-Marchesi, N., Costillo-Borrego, E., y Rivarosa, A. (2016). Análisis de guiones de laboratorio elaborados por docentes en formación de Argentina y España. In 27 Encuentros en Didáctica de las Ciencias Experimentales. Badajoz, 7 al 9 de noviembre de 2016.

Fernández-Marchesi, N., y Martínez-Aznar, M. M. (2017). Creencias de futuros profesores de secundario de física y química sobre las actividades experimentales. Enseñanza de Las Ciencias, (Extraordinario), 2601-2606.

Fernández-Marchesi, N., Martínez-Aznar, M. M., y Costillo-Borrego, E. (2017). Análisis comparativo de creencias sobre las actividades experimentales de futuros profesores de Biología y Geología. BioGrafía: Escritos Sobre La Biología y Su Enseñanza, (Extraordinario), 1333-1342.

Freitas, I., Jiménez, R., y Mellado, V. (2004). Solving physics problems: The conceptions and practice of an experienced teacher and an inexperienced teacher. Research in Science Education, 34(1), 113133.

Furió Más, C., Payá, J., y Valdés, P. (2005). ¿Cuál es el papel del trabajo experimental en la educación científica? In D. Gil Pérez, B. Macedo, J. Martínez Torregosa, C. Sifredo, P. Valdés, y A. Vilches (Eds.), ¿ Cómo promover el interés por la cultura científica ? Una propuesta didáctica fundamentada para la educación científica de jóvenes de 15 a 18 años. Santiago de Chile: UNESCO.

García Arteaga, E. (2011). Las prácticas experimentales en los textos y su influencia en el aprendizaje. Aporte histórico y filosófico en la física de campos. Universidad Autónoma de Barcelona, España.

García Arteaga, E., y Estany, A. (2010). Filosofía de las prácticas experimentales y enseñanza de las ciencias. Praxis Filosófica, (31), 7-24.

Garritz Ruiz, A., y Mellado, V. (2014). El conocimiento didáctico del contenido y la afectividad. In A. Garritz, S. Daza, y G. Lorenzo (Eds.), Conocimiento Didáctico del Contenido. Una perspectiva Iberoamericana. Saarbrücken, Alemania: Editorial Academia Española. 
Giraldo Mejía, J. A. (2016). El experimento en la enseñanza de la Biología. Un estudio de caso con licenciados en Biología de tres instituciones educativas distritales. Tesis de grado. Universidad Pedagógica Nacional.

Hacking, I. (1996). Representar e Intervenir. México D. F.: Paidós.

Hodson, D. (1994). Hacia un enfoque más crítico del trabajo de laboratorio. Enseñanza de Las Ciencias, 12(3), 299-313.

Iglesias, M. (2004). El giro hacia la práctica en filosofíade la ciencia: Una nueva perspectiva de la actividad experimental. Opción, 20(44), 98-119.

Insausti, M., y Merino, M. (2000). Una propuesta para el aprendizaje de contenidos procedimentales en el laboratorio de física y química. Investigações Em Ensino de Ciências, 5(2), 93-119.

Jacob, F. (1973). La lógica de lo viviente: una visión materialista de la biología. Barcelona: Laia.

Jiménez Tenorio, N., y Oliva, J. M. (2016). Aproximación al estudio de las estrategias didácticas en ciencias experimentales en formación inicial del profesorado de Educación Secundaria: descripción de una experiencia. Revista Eureka Sobre Enseñanza y Divulgación de Las Ciencias, 13(1), 121136.

Kirschner, P. (1992). Epistemology, Practical Work and Academic Skills in Science Education. Science y Education, 1(3), 273-299.

Litwin, E. (2000). Las configuraciones didácticas. Barcelona: Paidós.

Martínez-Aznar, M. M., y Ibáñez Orcajo, I. (2006). Resolver situaciones problemáticas en genética para modificar las actitudes relacionadas con la ciencia. Enseñanza de Las Ciencias, 24(2), 193-206.

Martínez-Aznar, M. M., Rodríguez-Arteche, I., y Gómez Lesarri, P. (2017). La resolución de problemas profesionales como referente para la formación inicial del profesorado de física y química. Revista Eureka Sobre Enseñanza y Divulgación de Las Ciencias, 14(1), 162-180.

Mayr, E. (1988). Hacia una nueva filosofía de la biología: Observaciones de un evolucionista. Harvard University Press.

Mayr, E. (1998). O Desenvolvimento do Pensamento Biológico. Diversidade, evolução e herança. Editora Universidade de Brasilia.

Mellado, V., Borrachero Cortés, A., Brígido Mero, M., Melo Niño, L., Dávila, M. A., Cañada Cañada, F., ... Bermejo, M. L. (2014). Las emociones en la enseñanza de las ciencias. Enseñanza de Las Ciencias, 32(3), 11-36.

Mellado, V., Ruiz, C., Bermejo, M. L., y Jiménez, R. (2006). Contributions from the philosophy of science to the education of science teachers. Science y Education, (15), 419-445.

Melo Niño, L., Cañada Cañada, F., Mellado, V., y Buitrago, A. (2016). Desarrollo del conocimiento didáctico del contenido en el caso de la enseñanza de la carga eléctrica en Bachillerato desde la práctica de aula. Revista Eureka Sobre Enseñanza y Divulgación de Las Ciencias, 13(2), 459-475.

Meredith, J., Fortneer, R., y Mullins, G. (1997). Model of affective learning for non formal science education facilities. Journal of Research in Science Teaching, 34(8), 805-818.

Miguens, M., y Garrett, R. (1991). Prácticas en la enseñanza de las ciencias. Problemas y posibilidades. Enseñanza de Las Ciencias, 9(3), 229-236.

Perrenoud, P. (2004). Desarrollar la práctica reflexiva en el oficio de enseñar. Profesionalización y razón pedagógica. México D. F.: Graó.

Porlán, R., y Martín del Pozo, R. (1996). Ciencia, profesores y enseñanza: unas relaciones complejas. Alambique. Didáctica de Las Ciencias Experimentales, 3(8), 23-32.

Porlán, R., Martín del Pozo, R., Rivero García, A., Harres, J., Azcárate, P., y Pizzato, M. (2010). El cambio del profesorado de ciencias I: marco teórico y formativo. Enseñanza de Las Ciencias, 28(1), 31-46.

Porlán, R., Martín Del Pozo, R., Rivero García, A., Harres, J., Azcárate, P., y Pizzato, M. (2011). El cambio del profesorado de ciencias II: Itinerarios de progresión y obstáculos en estudiantes de magisterio. Enseñanza de Las Ciencias, 29(3), 353-370.

Porlán, R., Rivero García, A., y Martín del Pozo, R. (1997). Conocimiento profesional y epistemología de los profesores I: Teoría, métodos e instrumentos. Enseñanza de Las Ciencias, 15(2), 155-171.

Porlán, R., Rivero García, A., y Martín del Pozo, R. (1998). Conocimiento profesional y epistemología de los profesores, II. Enseñanza de Las Ciencias, 16(2), 271-288.

Pozo, J. I., Pérez Echeverría, M., Martín Ortega, E., Scheuer Rubiños, N., de La Cruz, M., y Mateos Sanz, 
M. (2006). Nuevas formas de pensar la enseñanza y el aprendizaje: las concepciones de profesores y alumnos (Vol. 12). Barcelona: Grao.

Quesada Blázquez, M. Á. (2006). Es el momento de dar otro paso : De una filosofía del experimento hacia una filosofía de las prácticas científicas. In Congreso Iberoamericano de ciencia, tecnología, sociedad e innovación CTS. México.

Rivarosa, A., y Astudillo, C. (2013). Las prácticas científicas y la cultura: una reflexión necesaria para un educador de ciencias. Revista CTS, 8(23), 45-66.

Romero Chacón, Á., Morcillo Molina, C., García Arteaga, E., Tobón Cardona, E., Quinto Moya, J., Mejia Aristizábal, L., ... Aguilar Mosquera, Y. (2017). La experimentación en la clase de ciencias. Aportes a una enseñanza de las ciencias contextualizada con reflexiones metacientíficas. Medellín: Universidad de Antioquía.

Shulman, L. (1986). Those Who Understand: Knowledge Growth in Teaching. Educational Researcher, 15(2), 4-14.

Tobin, K., Tippins, D. J., y Gallard, A. J. (1994). Research on instructional strategies for teaching science. Handbook of Research on Science Education, 45, 93.

Vázquez Bernal, B., Jiménez Pérez, R., y Mellado, V. (2007). El desarrollo profesional del profesorado de Ciencias como integración de la reflexión y la práctica. La hipótesis de la complejidad. Revista Eureka Sobre Enseñanza y Divulgación de Las Ciencias, 4(3), 372-393. 


\section{ANEXO I}

Cuestionario sobre actividades experimentales

\section{Las clases de Laboratorio de Ciencias Naturales}

Este cuestionario pertenece a un proyecto de investigación sobre concepciones de los profesores de ciencias sobre las actividades experimentales. Agradecemos vuestra participación y garantizamos la privacidad de los datos.

a) mail

b) Nombre

c) Edad

d) Soy:

a. Profesor /profesora en ejercicio

b. Estudiante de profesorado

e) Titulación: En caso de ser estudiante, colocar la denominación del título que va a obtener

f) Año de finalización: En caso de ser estudiante colocar el año en que espera finalizar la carrera

g) ¿Cuánta experiencia tiene dando clases de nivel secundario? Puede colocar el dato en años o meses

h) ¿En qué colegio/ institución educativa trabaja? Si no trabaja en el sistema educativo colocas "no trabajo en el sistema"

i) ¿Tiene experiencia en otros niveles además de secundario?
a. Nivel primario
b. Universitario
c. Nivel inicial
d. Clases particulares
e. Formación de profesores de secundaria
f. Formación de profesores de primaria
g. Formación de profesores de nivel inicial
h. Otro:

j) Imagine que tiene que armar una clase de laboratorio a estudiantes de secundaria sobre un contenido de su disciplina (Biología, Física, Química, Geología).

a. Sobre que contenido de la asignatura lo haría

b. ¿Qué objetivos espera lograr con dicha actividad?

c. ¿Cuántas clases utilizaría?

d. ¿Cómo integraría esta actividad de laboratorio con sus clases de teoría en el aula?

k) ¿Cómo profesor que ventajas y desventajas ve en estas actividades prácticas de laboratorio con respecto a las clases de teoría?
a. Ventajas
b. Desventajas

I) Por favor redacte un guion de práctica de experimental que le daría a sus estudiantes (no se olvide de colocar todo lo que considera pertinente)

1 El término laboratorio húmedo (Wet Laboratory) en Biología, Química, Genética o Bioquímica, distingue los experimentos clásicos de laboratorio que manejan material biológico de los laboratorios secos (Dry Laboratory) en los que se realiza análisis por computadora u otro trabajo teórico.(Kirschner, 1992) 2 Se envió dos veces dado que la primera vez se recibieron escasas respuestas

3 La Asociación de Docentes de Ciencias Biológicas de la Argentina (ADBiA) es una organización que nuclea a profesores de Biología. Cuenta con más de 500 socios en todo el país y una trayectoria de más de 20 años en el país.

4 ADBiA tiene entre sus socios a profesores y a estudiantes de formación docente. Todos reciben el mismo boletín de la lista de correos electrónicos. Por ello se discriminaron ambos roles

5 Existen en el país, instituciones de educación superior públicas o privadas que ofrecen una postitulación de carácter docente para aquellos profesionales que no cuenten con dicha formación y que se encuentren dando clase. Dicho postítulo tiene una duración de dos años.

6 En Argentina, la formación de profesores tiene una duración de 4 años. Esto supone que si un egresado de la escuela secundaria (18 años) inicia sus estudios en el Instituto de Formación Docente, es esperable que a los 22/23 años haya egresado y comience a desempeñarse como docente.

7 En esta categoría, se analizaron las respuestas según los contenidos mencionados. En algunos casos un mismo docente señaló más de un contenido. 